\title{
Brain metastases in newly diagnosed colorectal cancer: a population-based study
}

This article was published in the following Dove Press journal: Cancer Management and Research

\section{Lin Yang ${ }^{1-3}$ \\ Wenzhuo $\mathrm{He}^{1-3}$ \\ Qiankun Xie ${ }^{1-3}$ \\ Shousheng $\mathrm{Liu}^{1-3}$ \\ Pengfei Kong ${ }^{1-3}$ \\ Chang Jiang ${ }^{1-3}$ \\ Bei Zhang ${ }^{1-3}$ \\ Liangping $\mathrm{Xia}^{1-3}$}

'Medical Oncology, Sun Yat-sen University Cancer Center, Guangzhou,

China; ${ }^{2}$ State Key Laboratory of

Oncology in Southern China, Guangzhou, China; ${ }^{3}$ Collaborative Innovation Center for Cancer Medicine, Guangzhou, China
Correspondence: Bei Zhang; Liangping $X_{i a}$

Medical Oncology, Sun Yat-sen University Cancer Center, 65I Dongfeng Road East, Guangzhou 510060, China

$\mathrm{Tel}+8602087343107$

Email zhangbei@sysucc.org.cn;

xilp@sysucc.org.cn
Background: Population-based incidence evaluations and prognosis assessments of brain metastasis (BM) at diagnosis of colorectal cancer (CRC) are lacking. Our study sought to determine the incidence of BM in CRC patients, median survival of patients with $\mathrm{BM}$, and the risk factors of BM in CRC.

Patients and methods: Patients diagnosed with CRC were identified using the Surveillance, Epidemiology, and End Results database. Multivariable logistic and Cox regression analyses were performed to identify predictors of the presence of BM at CRC diagnosis and the factors associated with poor survival. Kaplan-Meier analysis was used to estimate the survival difference between subgroups.

Results: We identified 170,793 adult patients diagnosed with CRC between 2010 and 2013. From these patients, we identified 401 patients with BM at the time of CRC diagnosis, which represents $0.23 \%$ of the entire patient CRC cohort and $1.3 \%$ of the patients with metastatic disease to any site. Median survival of patients with BM was 7.0 months, and the survival could increase to 15.59 months if there was no metastasis to other organs. We found that extracranial metastases number, tumor site, and pathology type were associated with BM at CRC diagnosis. Conclusion: The findings of this study indicate the incidence and prognosis for patients with $\mathrm{BM}$ at the time of CRC diagnosis. Our findings lend support for positive treatment for BM without metastasis to other organs.

Keywords: colorectal cancer, brain metastasis, tumor site, prognosis

\section{Introduction}

Approximately $20 \%$ of colorectal cancer (CRC) cases are found to have distant metastasis at the time of diagnosis, and the 5 -year survival rate is $<8 \% .^{1}$ The most common metastasis sites of CRC are the liver, lung, and peritoneum, but other metastatic sites have been described, such as bone, spleen, brain, and distant lymph nodes. ${ }^{2-4}$ A nationwide retrospective review from 3,827 autopsies generated insight into the metastatic patterns and demonstrated that primary cancers tend to metastasize differently with different frequencies to distinctive sites., ${ }^{2,5}$ Patients with rectal cancer more often metastasize to extra-abdominal sites, whereas colon cancer patients show a higher frequency of abdominal metastasis according to the pathological records of CRC patients. ${ }^{5}$

It is well known that the most common metastatic site for CRC patients is the liver, followed by the lung. ${ }^{2-4}$ However, the incidence of brain metastasis (BM) is very low $(1.2 \%-3.2 \%)^{6-9}$ and is of most concern because of neurological symptoms. Additionally, 
CRC with BM typically occurs at a later stage of disease progression. However, data regarding BM occurrence at the diagnosis of CRC are rare.

It has been reported that cases with uncommon metastases, including BM, are increasing. ${ }^{7}$ However, the overall survival (OS) of advanced CRC has been improving due to treatment progression. Cytotoxic agents and targeted therapies have improved the prognosis of unresectable CRC patients up to 30 months. ${ }^{10}$ Currently, the advancement of immunotherapy has markedly improved the prognosis of mismatch-repair deficient (microsatellite instability-deficient) cohort of CRC. ${ }^{11}$ Multidisciplinary treatments could be applied for the treatment of metastatic lesions, including the BM. ${ }^{12}$ Therefore, early diagnosis of BM is of vital importance. Unfortunately, the risk factors associated with the initial diagnosis of BM have not yet been fully investigated due to the limited number of patients.

It has been observed in clinical trial follow-ups that mucinous adenocarcinomas (MCs) have different distribution of metastatic disease compared with the more common adenocarcinoma (AC). Right-sided colon cancer (RCC) occurs in $\sim 10 \%-15 \%$ of CRC cases. RCC has often been considered a distinct entity, consisting of characteristics similar to $\mathrm{MC}$ with a poor prognosis in metastatic disease. ${ }^{13-15}$ However, whether these clinical factors contribute differently to $\mathrm{BM}$ in $\mathrm{CRC}$ remains unknown.

The purpose of the present study was to use the Surveillance, Epidemiology, and End Results (SEER) database to characterize the incidence of $\mathrm{BM}$ at the time of CRC diagnosis and examine if $\mathrm{BM}$ diagnosis is a risk factor for the prognosis of CRC. In addition, we sought to examine the clinical and sociodemographic predictors of poor survival among patients with $\mathrm{BM}$ at $\mathrm{CRC}$ diagnosis.

\section{Patients and methods}

Data regarding BM at the time of initial cancer diagnosis were retrieved from cases after 2010 in the SEER program of the National Cancer Institute SEER database. The data sets are available in the SEER data set repository https:// seer.cancer.gov/ and could present information on $30 \%$ of the US population.

The TNM stage was recorded as the SEER's “extent of disease" (for $\mathrm{T}$ and $\mathrm{M}$ stage) and the "number of positive nodes" (for N stage) coding schemes. Pathology was grouped as AC, MC, or other. Tumor grade was classified as well differentiated, moderately differentiated, poorly differentiated, or undifferentiated. Patients were stratified by CRC subtype, including RCC (cecum, ascending colon, hepatic flexure, and transverse colon), left-sided colon cancer (LCC; splenic flexure, descending colon, and sigmoid colon), rectosigmoid cancer (RSC; rectosigmoid junction and rectum), and appendix cancer. ${ }^{16}$ Race/ethnicity was categorized as previously described. Data on the presence of bone, lung, and liver metastases at diagnosis were available in the SEER database and were categorized as the extent of extracranial metastasis among patients in our study.

Patients were observed after their first diagnosis of CRC until the last follow-up, death, or end of the study, whichever occurred first.

Therefore, we included patients from 2010 to 2013, and patients who were proven to have BM 6 months after the diagnosis of CRC or were proven dead by autopsy were excluded. Thus, there were 170,793 patients diagnosed with CRC who were included in our analysis. Of these CRC patients, 401 were diagnosed with BM.

\section{Statistical analysis}

Multivariable logistic regression was used to determine which characteristic was associated with the presence of $\mathrm{BM}$ at diagnosis among CRC patients or the M1 cohort. Multivariable Cox regression was performed to identify the covariates associated with poor survival. The Kaplan-Meier method was used to estimate the survival difference between the subgroups.

Two-sided $P<0.05$ was considered statistically significant. Statistical analysis was performed using SAS 9.2 (SAS Institute, Cary, NC, USA).

\section{Ethics approval and consent to participate}

As the data used were from SEER data set, which is publicly available; ethics approval and consent to participate are not applicable.

\section{Results}

\section{Incidence of BM in patients diagnosed with CRC}

Among the 170,793 patients diagnosed with CRC, we demonstrated that $40.5 \%, 26.1 \%, 30.9 \%$, and $2.5 \%$ of patients had RCC, LCC, RSC, and appendix cancer, respectively. The number and incidence of patients with $\mathrm{CRC}$ and identified $\mathrm{BM}$ at $\mathrm{CRC}$ diagnosis are provided in Table 1, stratified by CRC cancer subtype. 
Table I Incidence and median survival of patients diagnosed with CRC and identified brain metastases at diagnosis

\begin{tabular}{|c|c|c|c|c|c|c|}
\hline \multirow[t]{2}{*}{ Subtype } & \multicolumn{3}{|l|}{ Patients (no.) } & \multicolumn{2}{|c|}{$\begin{array}{l}\text { Incidence proportion of brain } \\
\text { metastases, \% }\end{array}$} & \multirow{2}{*}{$\begin{array}{l}\text { Survival among } \\
\text { patients with brain } \\
\text { metastases, median } \\
\text { (IQR), months }\end{array}$} \\
\hline & With CRC & $\begin{array}{l}\text { With } \\
\text { metastatic } \\
\text { disease }\end{array}$ & $\begin{array}{l}\text { With brain } \\
\text { metastases }\end{array}$ & $\begin{array}{l}\text { Among the } \\
\text { entire cohort }\end{array}$ & $\begin{array}{l}\text { Among the subset } \\
\text { with metastatic } \\
\text { disease }\end{array}$ & \\
\hline Whole cohort & 170,793 & 31,544 & 401 & $0.2 \%$ & $1.3 \%$ & $21.95(21.87-22.03)$ \\
\hline $\mathrm{RCC}$ & $69,147(40.5 \%)$ & $|2,45|(\mid 8.0)$ & $167(0.2 \%)$ & $0.2 \%$ & $1.3 \%$ & $9.01(7.0-11.15)$ \\
\hline LCC & $44,642(26.1 \%)$ & 8,797 (19.7\%) & $92(0.2 \%)$ & $0.2 \%$ & $1.0 \%$ & $10.26(7.68-12.85)$ \\
\hline Rectosigmoid cancer & $52,784(30.9 \%)$ & $9,084(17.2 \%)$ & $139(0.3 \%)$ & $0.3 \%$ & $1.5 \%$ & I4.57 (II.89-17.26) \\
\hline Appendix cancer & $4,220(2.5 \%)$ & $\mathrm{I}, 2 \mathrm{I} 2$ (28.7\%) & $3(0.1 \%)$ & $0.1 \%$ & $0.3 \%$ & $5(0.84-9.16)$ \\
\hline \multicolumn{7}{|l|}{$\begin{array}{l}\text { Extracranial metastasis to } \\
\text { bone, lung, and liver (no.) }\end{array}$} \\
\hline 0 sites & & 145,175 & 110 & & $0.1 \%$ & 15.59 (11.60-19.59) \\
\hline Bone & & 357 & 12 & & $3.4 \%$ & $10.53(3.96-17.11)$ \\
\hline Lung & & 1,999 & 55 & & $2.8 \%$ & II .66 (8.35-I4.97) \\
\hline Liver & & 168,22 & 64 & & $0.4 \%$ & $10.52(7.02-14.02)$ \\
\hline Two sites & & 5,400 & 121 & & $2.2 \%$ & $8.85(7.12-10.57)$ \\
\hline All three sites & & 540 & 39 & & $6.6 \%$ & $7.34(4.82-9.86)$ \\
\hline
\end{tabular}

Abbreviations: CRC, colorectal cancer; LCC, left-sided colon cancer; RCC, right-sided colon cancer; IQR, interquartile range.

Among the entire cohort, 401 patients presented with BM, reflecting $0.23 \%$ of the entire study population and $1.3 \%$ of the subset with metastatic disease at any site. Incidence proportions were highest among patients with RCC $(0.2 \%$ of the entire cohort, $1.3 \%$ of the metastatic subset) and RSC $(0.3 \%$ of the entire cohort, $1.5 \%$ of the metastatic subset) subtypes.

Also, 3.4\% of the CRC patients with bone metastasis presented with BM at diagnosis, which was higher than that of liver or lung metastasis. Additionally, incidence proportions increased with extracranial metastatic sites $(6.6 \%$ for $\geq 3,2.2 \%$ for $2,0.7 \%$ for 1 , and $0.1 \%$ for 0 ). Interestingly, patients with liver metastasis demonstrated a low incidence rate $(0.4 \%)$ of $\mathrm{BM}$.

\section{Multivariable logistic regression}

On multivariable logistic regression (Table 2) among patients with metastatic cancer, T3-T4 stage patients vs Tis, T0, $\mathrm{T} 1$, and $\mathrm{T} 2(\mathrm{OR}=1.456,95 \% \mathrm{CI}, 1.048-2.022, P=0.025)$, $\mathrm{MC}$ vs $\mathrm{AC}(\mathrm{OR}=2.874,95 \% \mathrm{CI}, 1.385-5.967, P=0.005)$, tumor site (LCC vs RCC: OR $=0.756,95 \%$ CI, $0.581-0.983$, $P=0.037$; appendix vs RCC: $\mathrm{OR}=0.151,95 \% \mathrm{CI}, 0.04-0.488$, $P=0.002$ ), and metastatic disease to extracranial sites (bone vs none: $\mathrm{OR}=7.761,95 \% \mathrm{CI}, 5.372-11.214, P<0.001$; two sites vs none: $\mathrm{OR}=8.832,95 \% \mathrm{CI}, 3.484-22.386, P<0.001$; three vs none: $\mathrm{OR}=14.353,95 \% \mathrm{CI}, 9.311-22.125, P<0.001)$ were associated with significantly greater odds of presenting with BM at diagnosis. Age, gender, pathology grade, N stage, tumor size, pathology grade, race, or marital status was not associated with a higher risk of BM at CRC diagnosis using the multivariable model (Table 2).

\section{Survival}

The median survival among patients with BM (with metastasis to other organs) at diagnosis was 7.0 months (Figure 1A). As shown in Figure 1B, the median survival was 15.59 months (range 11.60-19.59 months) for patients with BM merely without metastasis to other organs (Figure 1B), which showed that patient prognosis could be improved if BM was diagnosed early. Then we compared survival difference of patients without metastasis, patients with BM only, patients with metastasis but without BM, and patients with metastasis including BM. Additionally, patients with multi-metastasis having shorter survival, and the reason maybe the $\mathrm{BM}$ as shown in Figure 1B (patients with metastasis without BM vs patients with metastasis including $\mathrm{BM}, P<0.001)$.

The median survival was 9.01, 10.26, 14.57, and 5 for RCC, LCC, RSC, and appendix cancer, respectively (Table 1). In the entire cohort of patients, RCC had the poorest survival and appendix cancer had the best survival $(P<0.001$; Figure 2A). However, in patients diagnosed with BM, RCC had the best survival $(P=0.002$; Figure $2 \mathrm{~B})$.

The multivariable Cox regression analysis of all patients is presented in Table 3. Age, T stage, N stage, pathology grade, pathology type, tumor sites, tumor size, race, marital status, extracranial metastatic number, and BM were all independent factors for poorer survival (all $P<0.001$ ). Multivariable Cox 
Table 2 Multivariable logistic regression for the presence of brain metastases at diagnosis of colorectal cancer

\begin{tabular}{|c|c|c|c|c|c|c|}
\hline \multirow[t]{2}{*}{ Variable } & \multicolumn{2}{|c|}{ Patients (no.) } & \multicolumn{2}{|l|}{$\begin{array}{l}\text { Among entire } \\
\text { cohort }\end{array}$} & \multicolumn{2}{|c|}{$\begin{array}{l}\text { Among the subset with } \\
\text { metastatic disease }\end{array}$} \\
\hline & $\begin{array}{l}\text { Number of } \\
\text { patients }\end{array}$ & $\begin{array}{l}\text { With brain } \\
\text { metastases }\end{array}$ & OR $(95 \% \mathrm{Cl})$ & $P$-value & OR $(95 \% \mathrm{Cl})$ & $P$-value \\
\hline Age at diagnosis (years) & & & & $P<0.00$ I & & $<0.001$ \\
\hline$<40$ & 5,239 & II (0.2\%) & I (reference) & & I (reference) & \\
\hline $40-49$ & 13,938 & $30(0.2 \%)$ & $1.045(0.519-2.103)$ & 0.902 & $\mathrm{I} .027(0.5 \mathrm{I} \mathrm{I}-2.066)$ & 0.939 \\
\hline $50-59$ & 35,039 & $112(0.3 \%)$ & $1.634(0.87 \mathrm{I}-3.064)$ & 0.126 & $1.754(0.936-3.285)$ & 0.079 \\
\hline $60-69$ & 42,210 & $|2|(0.3 \%)$ & $1.402(0.748-2.629)$ & 0.292 & I.473 (0.787-2.755) & 0.226 \\
\hline$>70$ & 74,078 & $127(0.2 \%)$ & $0.853(0.454-1.603)$ & 0.621 & $0.963(0.5 \mid 4-1.805)$ & 0.907 \\
\hline Sex & & & & NA & & NA \\
\hline Male & 88,925 & $206(0.2 \%)$ & I (reference) & NA & I (reference) & NA \\
\hline Female & 81,467 & $195(0.2 \%)$ & NA & NA & NA & NA \\
\hline T stage & & & & 0.005 & & 0.007 \\
\hline Tis, T0, TI, T2 $(0, \mathrm{I}, 2,3)$ & $55,5 \mid 4$ & $65(0.1 \%)$ & I (reference) & & I (reference) & \\
\hline T3-T4 $(4,5)$ & 96,802 & $186(0.2 \%)$ & $1.240(0.899-1.710$ & 0.189 & $1.456(1.048-2.022)$ & 0.025 \\
\hline Tx & 18,076 & $150(0.8 \%)$ & 1.695 (1.224-2.347) & 0.001 & $1.085(0.803-1.467)$ & 0.594 \\
\hline N stage & & & & 0.030 & & NA \\
\hline No & 98,168 & $134(0.1 \%)$ & I (reference) & & I (reference) & NA \\
\hline NI & 39,027 & $118(0.3 \%)$ & $1.408(1.053-1.882)$ & 0.021 & NA & NA \\
\hline N2 & 21,348 & $76(0.4 \%)$ & $\mathrm{I} .838(\mathrm{I} .202-2.8 \mathrm{II})$ & 0.005 & NA & NA \\
\hline $\mathrm{Nx}$ & 11,849 & $73(0.6 \%)$ & $1.273(0.923-1.756)$ & 0.141 & NA & NA \\
\hline Pathology grade & & & & NA & & 0.050 \\
\hline Well differentiated & 16,648 & $8(0.0 \%)$ & I (reference) & NA & I (reference) & \\
\hline Moderately differentiated & 102,478 & $160(0.2 \%)$ & NA & NA & $0.581(0.384-0.880)$ & 0.010 \\
\hline Poorly differentiated & 23,548 & $100(0.4 \%)$ & NA & NA & $1.006(0.635-1.596)$ & 0.978 \\
\hline Undifferentiated & 4,588 & $16(0.3 \%)$ & NA & NA & $1.355(0.745-2.463)$ & 0.320 \\
\hline Unknown & 23,130 & $117(0.5 \%)$ & $3.134(1.516-6.480)$ & 0.002 & $5.149(2.475-10.713)$ & $<0.001$ \\
\hline Pathology type & & & & $<0.001$ & & 0.005 \\
\hline Adenocarcinoma & $|5|, 257$ & $339(0.2 \%)$ & I (reference) & & I (reference) & \\
\hline Mucinous carcinoma & 13,983 & $27(0.2 \%)$ & $2.738(1.336-5.613)$ & 0.006 & $2.874(1.385-5.967)$ & 0.005 \\
\hline Other type ${ }^{\mathrm{a}}$ & 3,932 & $22(0.6 \%)$ & $2.011(0.968-4.176)$ & 0.061 & $1.898(0.927-3.888)$ & 0.089 \\
\hline Unknown & 1,220 & $13(1.1 \%)$ & $4.813(2.023-\mid \mathrm{I} .452)$ & $<0.001$ & $2.660(1.119-6.323)$ & 0.027 \\
\hline Tumor site & & & & 0.004 & & 0.003 \\
\hline $\mathrm{RCC}$ & 68,980 & $167(0.2 \%)$ & I (reference) & & & \\
\hline LCC & 44,550 & $92(0.2 \%)$ & $0.766(0.588-0.996)$ & 0.047 & $0.756(0.58 I-0.983)$ & 0.037 \\
\hline Rectosigmoid & 52,645 & $139(0.3 \%)$ & $0.657(0.5 \mid 3-0.842)$ & 0.001 & $0.808(0.633-1.033)$ & 0.089 \\
\hline Appendix & 4,217 & $3(0.1 \%)$ & 0.377 (0.1 I9-1.199) & 0.098 & $0.151(0.04-0.488)$ & 0.002 \\
\hline Tumor size (mm) & & & & NA & & NA \\
\hline$\leq 40$ & 69,456 & $8 \mathrm{I}(0.1 \%)$ & I (reference) & NA & I (reference) & NA \\
\hline $40-70$ & 48,304 & $114(0.2 \%)$ & NA & NA & NA & NA \\
\hline$\geq 70$ & 18,304 & $47(0.3 \%)$ & NA & NA & NA & NA \\
\hline Unknown & 34,328 & $159(0.5 \%)$ & NA & NA & NA & NA \\
\hline Race & & & & 0.088 & & NA \\
\hline Hispanic & 20,436 & $46(0.2 \%)$ & I (reference) & & I (reference) & NA \\
\hline Black & II5,273 & $280(0.2 \%)$ & $0.702(0.5 \mathrm{I} I-0.966)$ & 0.030 & NA & NA \\
\hline White & $|3,84|$ & $22(0.2 \%)$ & $1.005(0.735-1.375)$ & 0.973 & NA & NA \\
\hline Asian/Pacific Islander & 1,222 & 0 & $0.620(0.399-0.962)$ & 0.033 & NA & NA \\
\hline Native American/Alaska Native & 1,217 & $5(0.4 \%)$ & $1.439(0.583-3.547)$ & 0.990 & NA & NA \\
\hline Unknown & $\mathrm{I}, 144$ & 0 & $0.000(0.000-)$ & 0.990 & NA & NA \\
\hline Marital status & & & & NA & & NA \\
\hline Unmarried & 71,525 & $184(0.3 \%)$ & I (reference) & NA & I (reference) & NA \\
\hline Married & 87,779 & $199(0.2 \%)$ & NA & NA & NA & NA \\
\hline Unknown & 11,088 & $18(0.2 \%)$ & NA & NA & NA & NA \\
\hline $\begin{array}{l}\text { Extracranial metastasis to } \\
\text { bone, lung, and liver (no.) }\end{array}$ & & & & $<0.001$ & & $<0.001$ \\
\hline 0 sites & 145,065 & $110(0.1 \%)$ & I (reference) & & I (reference) & \\
\hline Bone & 19,047 & $|3|(0.7 \%)$ & $19.212(10.264-35.964)$ & $<0.001$ & $7.761(5.372-11.214)$ & $<0.001$ \\
\hline
\end{tabular}


Table 2 (Continued)

\begin{tabular}{|c|c|c|c|c|c|c|}
\hline \multirow[t]{2}{*}{ Variable } & \multicolumn{2}{|c|}{ Patients (no.) } & \multicolumn{2}{|l|}{$\begin{array}{l}\text { Among entire } \\
\text { cohort }\end{array}$} & \multicolumn{2}{|c|}{$\begin{array}{l}\text { Among the subset with } \\
\text { metastatic disease }\end{array}$} \\
\hline & $\begin{array}{l}\text { Number of } \\
\text { patients }\end{array}$ & $\begin{array}{l}\text { With brain } \\
\text { metastases }\end{array}$ & OR (95\% Cl) & $P$-value & OR (95\% Cl) & $P$-value \\
\hline Lung & & & $21.320(15.045-30.212)$ & $<0.001$ & $1.306(0.690-2.470)$ & 0.413 \\
\hline Liver & & & $2.572(1.846-3.583)$ & $<0.001$ & $1.327(0.944-1.866)$ & 0.171 \\
\hline Two sites & 5,279 & $|2|(2.2 \%)$ & $12.780(9.469-17.249)$ & $<0.001$ & 8.832 (3.484-22.386) & $<0.001$ \\
\hline All three sites & 506 & $34(6.3 \%)$ & $34.847(22.767-3.336)$ & $<0.001$ & $14.353(9.311-22.125)$ & $<0.001$ \\
\hline Unknown & 495 & $5(1.0 \%)$ & $6.922(2.780-17.236)$ & $<0.001$ & $1.510(0.604-3.774)$ & 0.377 \\
\hline
\end{tabular}

Note: ${ }^{2}$ The pathology type other than adenocarcinoma or mucinous carcinoma. Bold values indicate the $P$ value is significant when two-sided $P<0.05$ was considered statistically significant.

Abbreviations: LCC, left-sided colon cancer; NA, not available; RCC, right-sided colon cancer.
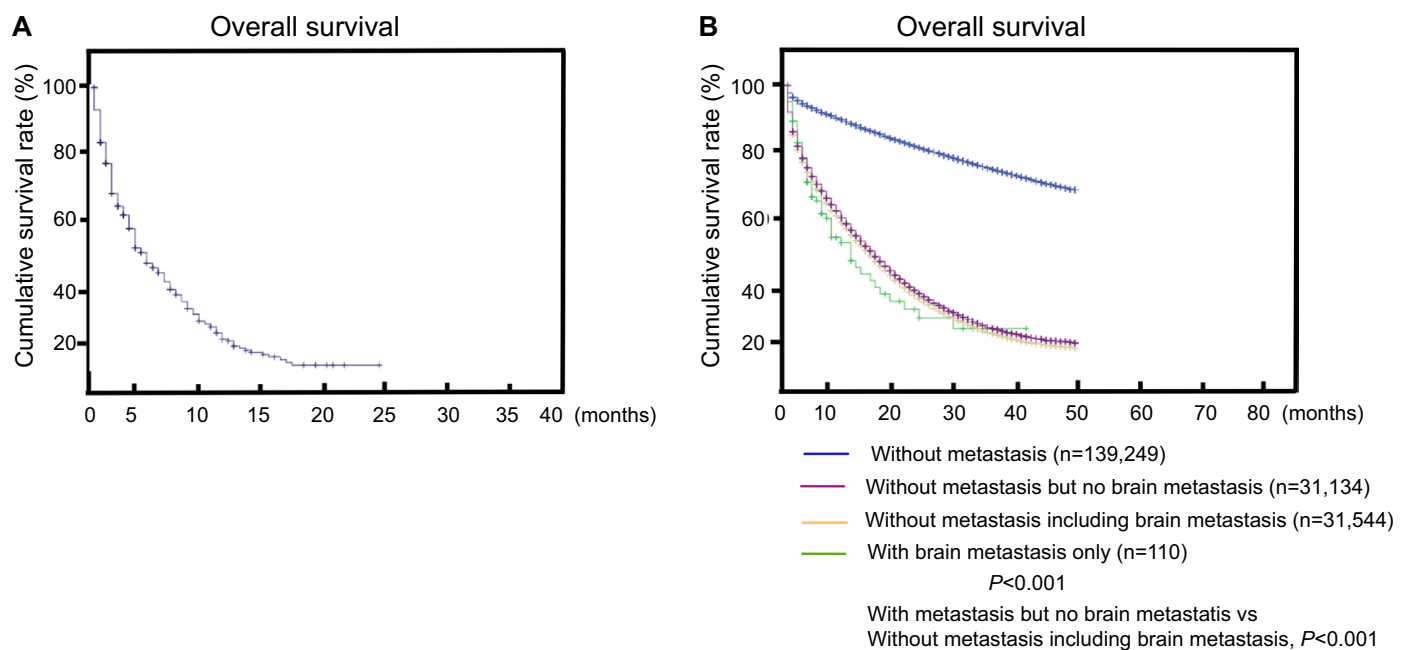

Figure I (A) Overall survival among patients with BM. (B) Survival stratified by subtype (nonmetastatic patients, patients with only BM, patients with metastasis to other sites but no BM, and patients with metastasis to multiple sites including BM).

Abbreviation: BM, brain metastasis.

regression analysis also showed that among patients with $\mathrm{BM}$ at $\mathrm{CRC}$ diagnosis, age, pathology type (MC vs $\mathrm{AC}$, $\mathrm{HR}=1.146,95 \% \mathrm{CI}, 1.062-1.237, P<0.001$ ), tumor site (RSC vs $\mathrm{RCC}, \mathrm{HR}=0.661,95 \% \mathrm{CI}, 0.491-0.890, P<0.001)$, and extracranial metastatic number ( 2 vs $0 / 1, H R=1.471,95 \%$ CI, $1.127-1.920, P<0.001$; all 3 vs $0 / 1, \mathrm{HR}=1.686,95 \% \mathrm{CI}$, $1.102-2.579, P=0.016)$ were significantly associated with poorer survival (Table 3 ).

OS estimates by the extent of extracranial metastatic disease is shown is Figure 2C, D. In general, survival was poorer among patients who displayed more extensive systemic disease at CRC diagnosis. However, this trend was merely significant in the cohort of extracranial systemic disease only ( 3 sites vs 2 sites, $P<0.001$; 2 sites vs 1 site, $P<0.001$; Figure $2 \mathrm{E}$ ) and was not found in the BM cohort ( 2 sites vs 1 site, $P=0.559$; 3 sites vs 2 sites, $P=0.322$; Figure $2 \mathrm{~F}$ ).
Table 4 displays the median survival by subtype as stratified by the extent of systemic disease in the cohort of extracranial systemic disease only and the cohort of extracranial systemic disease and BM. In addition, we found that the presence of BM at initial diagnosis was associated with a shorter survival time compared with patients who presented with de novo metastatic disease without baseline brain involvement (Table 4).

\section{Discussion}

In the present study, the median OS for patients with BM (with metastasis to other organs) was 7 months, which was shorter than reported previously ( 14.5 months). ${ }^{17}$ The median survival was 15.59 months (range 11.60-19.59 months) for patients with BM merely without metastasis to other organs. Recent studies have proved that the OS in CRC patients has 
A

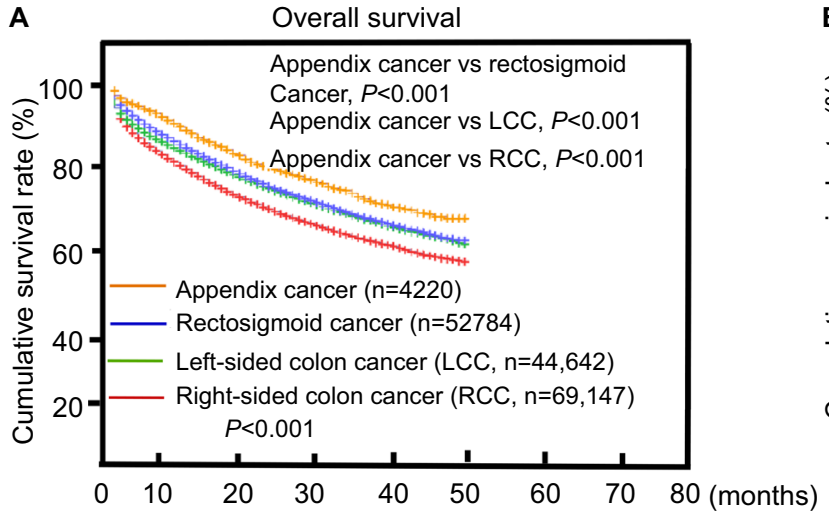

C

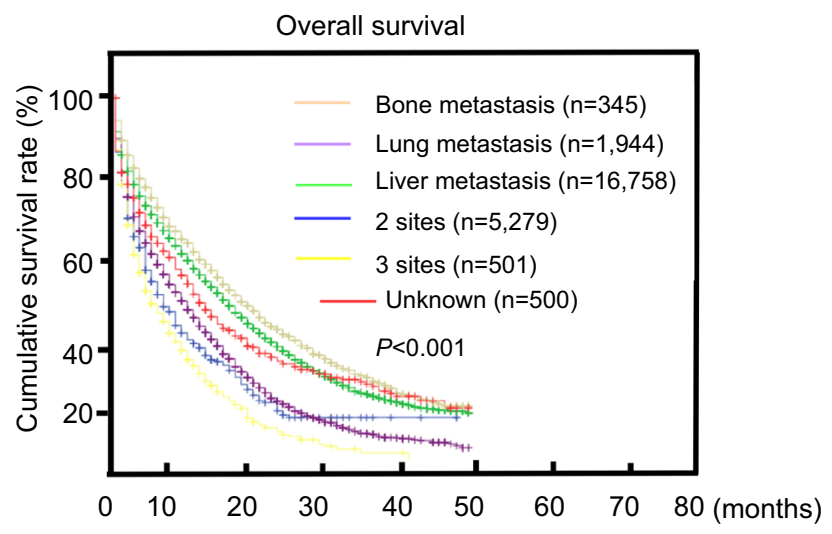

E

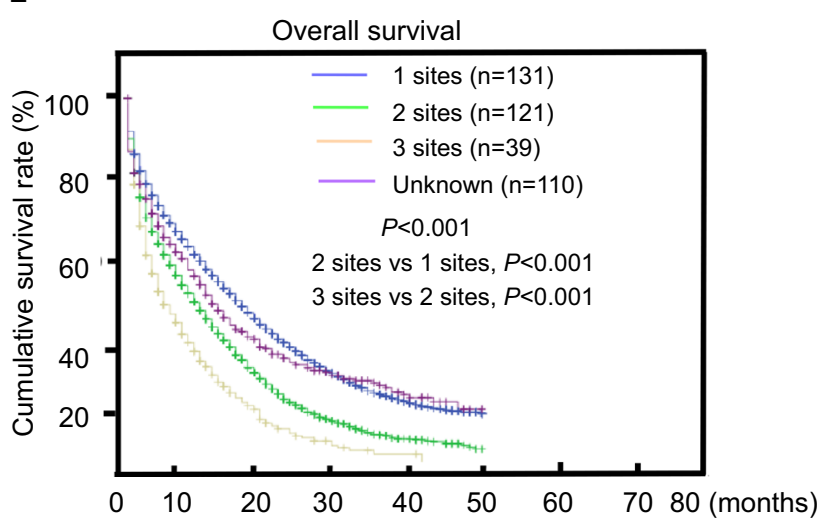

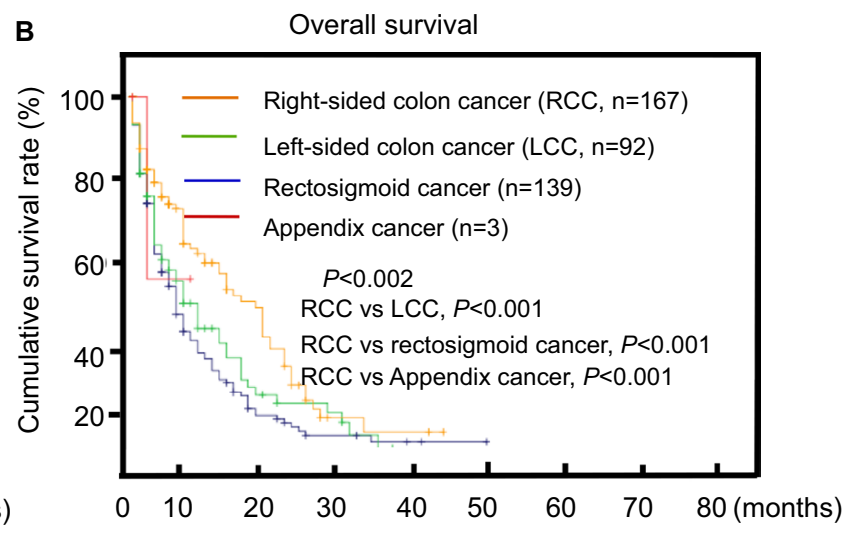

D

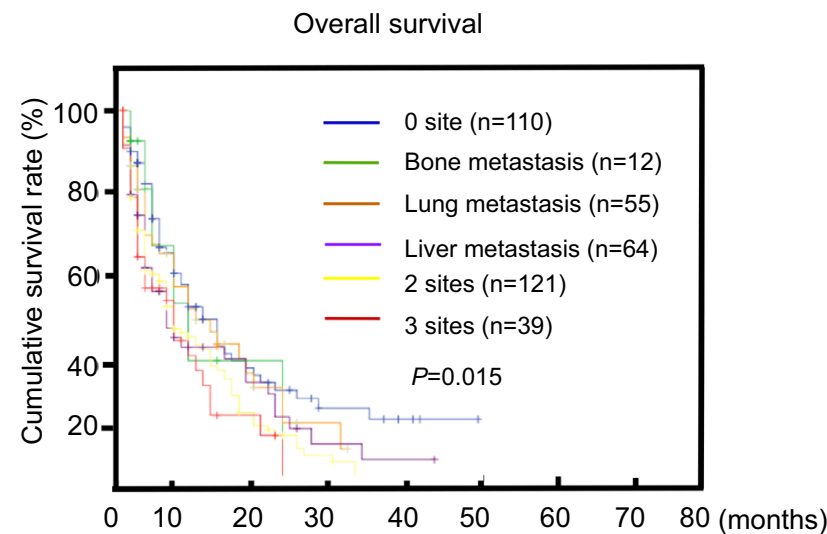

$\mathbf{F}$

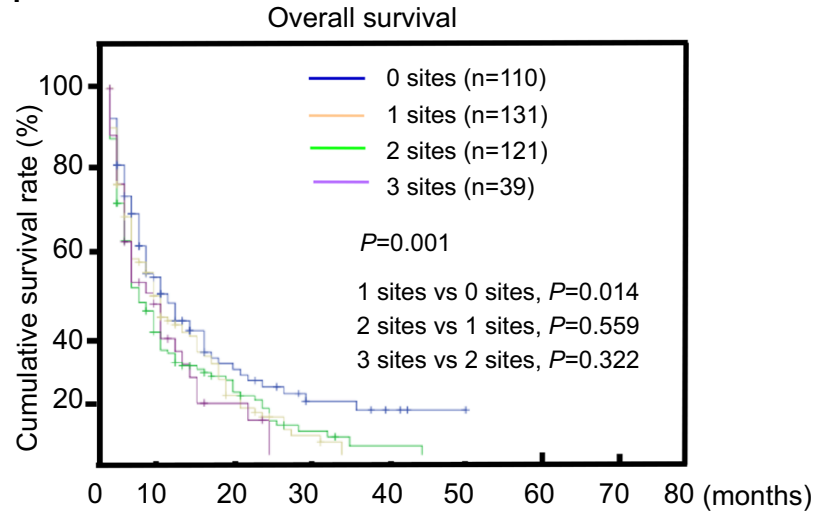

Figure 2 Overall survival among patients with colorectal cancer and brain metastases at diagnosis.

Notes: Survival stratified by tumor site (RCC, LCC, rectosigmoid cancer, appendix cancer) (A) in the whole cohort or (B) in patients with BM. Survival stratified by the metastasis organ and the extent of extracranial metastatic disease in the (C) cohort of extracranial systemic disease only and the (D) brain metastasis cohort. Extent of extracranial metastatic disease is classified by the number of metastatic sites to the bone, lung, or liver in the (E) cohort of extracranial systemic disease only and (F) the brain metastasis cohort.

Abbreviations: LCC, left-sided colon cancer; RCC, right-sided colon cancer.

improved since the late $1990 \mathrm{~s},{ }^{18,19}$ therefore, new analyses are needed to focus on treatment for BM in patients diagnosed with CRC, especially for the patients with BM merely without metastasis to other organs.
We demonstrated that tumor site, bone metastases, and more number of extracranial metastasis were associated with $\mathrm{BM}$ at $\mathrm{CRC}$ diagnosis. Sundermeyer et al reported that lung metastasis is a risk factor for BM; the incidence is higher in 
Table 3 Multivariable Cox regression for overall survival among patients with brain metastases or among metastatic disease subsets

\begin{tabular}{|c|c|c|c|c|}
\hline \multirow[t]{2}{*}{ Variable } & \multicolumn{2}{|c|}{$\begin{array}{l}\text { Among the subset with } \\
\text { metastatic disease }\end{array}$} & \multicolumn{2}{|l|}{$\begin{array}{l}\text { Among the brain } \\
\text { metastasis cohort }\end{array}$} \\
\hline & HR (95\% Cl) & $P$-value & HR (95\% Cl) & $P$-value \\
\hline Age at diagnosis (years) & & $<0.001$ & & $<0.001$ \\
\hline$<40$ & I (reference) & & I (reference) & \\
\hline $40-49$ & $1.004(0.915-1.102)$ & 0.928 & 0.681 (0.264-I.753) & 0.425 \\
\hline $50-59$ & $1.175(1.078-1.280)$ & $<0.001$ & 1.161 (0.525-2.564) & 0.712 \\
\hline $60-69$ & I.4II (I.297-I.535) & $<0.001$ & $1.832(0.836-4.014)$ & $0 .|3|$ \\
\hline$>70$ & 2.171 (1.997-2.359) & $<0.001$ & $2.660(1.212-5.840)$ & 0.015 \\
\hline Sex & & NA & & NA \\
\hline Male & I (reference) & & I (reference) & \\
\hline Female & NA & NA & NA & NA \\
\hline T stage & & $<0.001$ & & NA \\
\hline Tis, T0, TI, T2 (0, I, 2, 3) & I (reference) & & I (reference) & \\
\hline T3-T4 $(4,5)$ & $1.018(0.970-1.069)$ & 0.469 & NA & NA \\
\hline Tx & 1.099 (1.048-I.I53) & $<0.001$ & NA & NA \\
\hline N stage & & $<0.001$ & & NA \\
\hline No & I (reference) & & I (reference) & \\
\hline $\mathrm{NI}$ & $0.926(0.887-0.967)$ & 0.001 & NA & NA \\
\hline N2 & $1.090(1.028-1.155)$ & 0.004 & NA & NA \\
\hline $\mathrm{Nx}$ & $1.082(1.032-1.134)$ & 0.001 & NA & NA \\
\hline Pathology grade & & $<0.001$ & & NA \\
\hline Well differentiated & I (reference) & & I (reference) & \\
\hline Moderately differentiated & $1.723(1.59 \mid-1.865)$ & $<0.001$ & NA & NA \\
\hline Poorly differentiated & $1.845(1.668-2.04 I)$ & $<0.001$ & NA & NA \\
\hline Undifferentiated & $2.577(0.858-7.740)$ & 0.092 & NA & NA \\
\hline Unknown & $1.584(0.605-4.146)$ & 0.349 & NA & NA \\
\hline Pathology type & & $<0.001$ & & $<0.001$ \\
\hline Adenocarcinoma & I (reference) & & I (reference) & \\
\hline Mucinous carcinoma & 1.102 (1.048-I.I59) & $<0.001$ & $1.146(1.062-1.237)$ & $<0.001$ \\
\hline Other type $\mathrm{a}^{\mathrm{a}}$ & $1.613(1.5 \mid 2-1.721)$ & $<0.001$ & $0.883(0.337-2.315)$ & 0.800 \\
\hline Unknown & $2.269(2.083-2.47 I)$ & $<0.001$ & $1.185(0.450-3.123)$ & 0.731 \\
\hline Tumor site & & $<0.001$ & & 0.029 \\
\hline $\mathrm{RCC}$ & I (reference) & & I (reference) & \\
\hline LCC & $0.813(0.786-0.842)$ & $<0.001$ & $0.984(0.722-1.342)$ & 0.919 \\
\hline Rectosigmoid & $0.654(0.63 I-0.679)$ & $<0.001$ & $0.66 \mathrm{I}(0.49 \mathrm{I}-0.890)$ & 0.006 \\
\hline Appendix & $0.423(0.38 I-0.470)$ & $<0.001$ & $0.372(0.047-2.970)$ & 0.351 \\
\hline Tumor size & & $<0.001$ & & NA \\
\hline$\leq 40$ & I (reference) & & I (reference) & \\
\hline $40-70$ & I.I 22 (I.077-I.I68) & $<0.001$ & NA & NA \\
\hline$\geq 70$ & $1.278(1.218-1.342)$ & $<0.001$ & NA & NA \\
\hline Unknown & $1.182(1.131-1.235)$ & $<0.001$ & NA & NA \\
\hline Race & & $<0.001$ & & NA \\
\hline Hispanic & I (reference) & & I (reference) & \\
\hline Black & $1.117(1.073-1.163)$ & $<0.001$ & NA & NA \\
\hline White & $1.013(0.966-1.061)$ & 0.599 & NA & NA \\
\hline Asian/Pacific Islander & $0.946(0.896-1.000)$ & 0.050 & NA & NA \\
\hline Native American/Alaska Native & $1.273(1.086-1.49 \mid)$ & 0.003 & NA & NA \\
\hline Unknown & $0.712(0.459-1.105)$ & 0.130 & NA & NA \\
\hline Marital status & & $<0.001$ & NA & NA \\
\hline Unmarried & I (reference) & & NA & NA \\
\hline Married & $0.802(0.779-0.826)$ & $<0.001$ & NA & NA \\
\hline Unknown & $0.849(0.796-0.907)$ & $<0.001$ & NA & NA \\
\hline Extracranial metastasis to bone, & & $<0.001$ & & 0.004 \\
\hline lung, and liver (no.) & & & & \\
\hline $0 / 1$ & I (reference) & & & \\
\hline 2 & $1.109(1.066-1.153)$ & $<0.001$ & 1.471 (1.127-1.920) & 0.004 \\
\hline
\end{tabular}


Table 3 (Continued)

\begin{tabular}{|l|l|l|l|l|}
\hline Variable & \multicolumn{2}{|l|}{\begin{tabular}{l} 
Among the subset with \\
\multicolumn{2}{l|}{}
\end{tabular}} & \multicolumn{2}{l|}{$\begin{array}{l}\text { Among the brain } \\
\text { metastasis cohort }\end{array}$} \\
\cline { 2 - 5 } & HR (95\% Cl) & P-value & HR (95\% Cl) & $P$-value \\
\hline All three & $1.423(1.356-1.494)$ & $<0.001$ & $1.686(1.102-2.579)$ & 0.016 \\
Unknown & $1.854(1.673-2.054)$ & $<0.00 I$ & & \\
Brain metastasis (yes vs no) & $1.360(1.203-1.536)$ & $<0.00 I$ & & \\
\hline
\end{tabular}

Note: aThe pathology type other than adenocarcinoma or mucinous carcinoma.

Abbreviations: LCC, left-sided colon cancer; NA, not available; RCC, right-sided colon cancer.

Table 4 Median survival of colorectal cancer patients by the extent of systemic metastatic disease

\begin{tabular}{|l|l|l|}
\hline Subtype & Survival, median, months & \multicolumn{1}{l|}{$\begin{array}{l}\text { Extracranial systemic } \\
\text { disease and brain metastases }\end{array}$} \\
\cline { 2 - 3 } & $\begin{array}{l}\text { Extracranial systemic } \\
\text { disease only }\end{array}$ & \\
\hline Extracranial metastasis to bone, lung, and liver or & & $10.53(3.96-17.11)$ \\
number of metastasis sites & $23.91(22.81-25.01)$ & $10.52(7.02-14.02)$ \\
Bone & $14.36(13.86-14.86)$ & $11.66(8.35-14.97)$ \\
Lung & $21.21(20.86-21.57)$ & $8.85(7.13-10.97)$ \\
Liver & $13.93(11.76-16.10)$ & $7.34(4.82-9.86)$ \\
Two sites & $9.51(8.41-10.62)$ & $(4.82)$ \\
All three sites &
\end{tabular}

lung metastasis than with liver and peritoneal metastases. ${ }^{7}$ Many studies have found that metastasis progresses sequentially in many patients, and that BM is one of the late sites of involvement. ${ }^{20,21}$ RCC showed more frequency of BM than any other tumor site among all the BM patients. Furthermore, we found that RCC was an independent risk factor of BM. Previous studies have shown that RCC may be a different entity from LCC, including differences in epidemiological, clinical, and molecular biological, and prognostic findings between RCC and LCC. ${ }^{22-24}$ Additionally, we found that $\mathrm{RCC}$ had the poorest survival. However, when patients were diagnosed with BM, RCC patients had better survival than LCC patients and appendix cancer had the poorest survival. We are unsure of the mechanism of this phenomenon.

In addition, age and pathology type were independent factors in patients with BM at the diagnosis of CRC (in the whole cohort and the M1 cohort). Additionally, patients presenting with appendix tumors with BM, of age $>70$ years, and with MC showed the poorest survival. Age at the time of initial diagnosis was the variable that constantly revealed a significant effect on survival, suggesting a better performance status in younger patients. ${ }^{25-28}$ Major immune defects occur at an older age, including the lack of naïve T-cells, impaired activation pathways of T-cells, antigen-presenting cells, and age-related changes in the tumor microenvironment. Patients with $\mathrm{MC}$ showed poorer survival than patients with $\mathrm{AC}$ in
CRC diagnosed with BM. The presence of metastatic disease in more than one location was more frequent in MC compared with AC..$^{5}$ In MC patients, we found a high rate of peritoneal metastases. Several clinical studies have suggested differences in metastatic patterns between histological subtypes. ${ }^{13,29,30}$ Since curative surgery is an option that is mainly limited to liver metastases, this may be an explanation for the poor performance of MC patients in trials for metastatic disease. ${ }^{13,29}$

In the current study, the unadjusted probability of BM was calculated as 3.4\% for CRC metastases. The incidence of BM has increased as much as $3 \%$ since recent extended treatment options have been made available, which have caused an improvement in advanced CRC prognosis. ${ }^{7}$ Therefore, the potential of BM should be made aware to the clinicians, especially when patients present with RCC, have bone metastases, or more than three extracranial metastases. For such high-risk patients, brain scans may have to be considered.

Limitations of this study include its retrospective design, selection bias, and heterogeneous population. Furthermore, in the CRC patients, brain imaging was not routinely performed, except at the occurrence of symptoms, which may potentially underestimate the true positive rate of BM. In addition, our patients were not analyzed for the effects of gene mutation in BM tumors at the initial diagnosis, although a recent study demonstrated that RAS- or PIK3CA-mutant CRC patients were more likely to develop BM. ${ }^{31}$ 
In this study we found that RCC, MC, bone metastases, and more than three extracranial metastases were independent predictors of BM in CRC patients. People with BM without metastasis to other organs had relatively better survival than BM patients with metastasis to other organs. So, it is important to improve available therapeutic options to enhance quality of life, especially for the people with BM without metastasis to other organs.

\section{Data sharing statement}

All data were retrieved from the Surveillance, Epidemiology, and End Results (SEER) program of the National Cancer Institute between 2010 and 2012. The data sets are available in the SEER data set repository https://seer.cancer.gov/.

\section{Acknowledgments}

We would like to thank the native English-speaking scientists of Elixigen Company (Huntington Beach, CA, USA) for editing our manuscript. This work was supported by the Natural Science Foundation of Guangdong, China (grant number 2015A030313010).

\section{Author contributions}

Conceptualization: YL, ZB, and XLP; methodology: YL and HWZ; software and data curation: YL, HWZ, and XQK; formal analysis, LSS, KPF, and JC; revising the article critically for important intellectual content: XLP; writing-original draft preparation: $\mathrm{YL}$; project administration and funding acquisition: XLP. All authors (YL, HWZ, XQK, LSS, KPF, JC, ZB, and XLP) have read and approved the final manuscript. All authors contributed to data analysis, drafting and revising the article, gave final approval of the version to be published, and agree to be accountable for all aspects of the work.

\section{Disclosure}

The authors report no conflicts of interest in this work.

\section{References}

1. Cook AD, Single R, Mccahill LE. Surgical resection of primary tumors in patients who present with stage IV colorectal cancer: an analysis of surveillance, epidemiology, and end results data, 1988 to 2000. Ann Surg Oncol. 2005;12(8):637-645.

2. Disibio G, French SW. Metastatic patterns of cancers: results from a large autopsy study. Arch Pathol Lab Med. 2008;132(6):931-939.

3. Hess KR, Varadhachary GR, Taylor SH, et al. Metastatic patterns in adenocarcinoma. Cancer. 2006;106(7):1624-1633.

4. Weiss L, Grundmann E, Torhorst J, et al. Haematogenous metastatic patterns in colonic carcinoma: an analysis of 1541 necropsies. J Pathol. 1986;150(3):195-203.

5. Hugen N, van de Velde CJ, de Wilt JH, Nagtegaal ID. Metastatic pattern in colorectal cancer is strongly influenced by histological subtype. Ann Oncol. 2014;25(3):651-657.
6. Ko FC, Liu JM, Chen WS, Chiang JK, Lin TC, Lin JK. Risk and patterns of brain metastases in colorectal cancer: 27-year experience. Dis Colon Rectum. 1999;42(11):1467-1471.

7. Sundermeyer ML, Meropol NJ, Rogatko A, Wang H, Cohen SJ. Changing patterns of bone and brain metastases in patients with colorectal cancer. Clin Colorectal Cancer. 2005;5(2):108-113.

8. Nieder C, Pawinski A, Balteskard L. Colorectal cancer metastatic to the brain: time trends in presentation and outcome. Oncology. 2009;76(5):369-374.

9. Noura $\mathrm{S}$, Ohue M, Shingai T, et al. Brain metastasis from colorectal cancer: prognostic factors and survival. J Surg Oncol. 2012;106(2): 144-148.

10. Jawed I, Wilkerson J, Prasad V, Duffy AG, Fojo T. Colorectal cancer survival gains and novel treatment regimens: a systematic review and analysis. JAMA Oncol. 2015;1(6):787-795.

11. Asaoka Y, Ijichi H, Koike K. PD-1 blockade in tumors with mismatchrepair deficiency. $N$ Engl J Med. 2015;373(20):1979.

12. Soffietti R, Rudà R, Trevisan E. Brain metastases: current management and new developments. Curr Opin Oncol. 2008;20(6):676-684.

13. Mekenkamp LJ, Heesterbeek KJ, Koopman M, et al. Mucinous adenocarcinomas: poor prognosis in metastatic colorectal cancer. Eur J Cancer. 2012;48(4):501-509.

14. Hyngstrom JR, Hu CY, Xing Y, et al. Clinicopathology and outcomes for mucinous and signet ring colorectal adenocarcinoma: analysis from the National Cancer Data Base. Ann Surg Oncol. 2012;19(9): 2814-2821.

15. Hugen N, Verhoeven RH, Radema SA, et al. Prognosis and value of adjuvant chemotherapy in stage III mucinous colorectal carcinoma. Ann Oncol. 2013;24(11):2819-2824.

16. Rabeneck L, Davila JA, El-Serag HB. Is there a true "shift" to the right colon in the incidence of colorectal cancer? Am J Gastroenterol. 2003;98(6):1400-1409.

17. Michl M, Thurmaier J, Schubert-Fritschle G, et al. Brain metastasis in colorectal cancer patients: survival and analysis of prognostic factors. Clin Colorectal Cancer. 2015;14(4):281-290.

18. Schwartzberg LS, Rivera F, Karthaus M, et al. PEAK: a randomized, multicenter Phase II study of panitumumab plus modified fluorouracil, leucovorin, and oxaliplatin (mFOLFOX6) or bevacizumab plus mFOLFOX6 in patients with previously untreated, unresectable, wild-type KRAS exon 2 metastatic colorectal cancer. J Clin Oncol. 2014;32(21):2240-2247.

19. Stintzing S, Modest DP, Rossius L, et al. FOLFIRI plus cetuximab versus FOLFIRI plus bevacizumab for metastatic colorectal cancer (FIRE-3): a post-hoc analysis of tumour dynamics in the final RAS wild-type subgroup of this randomised open-label Phase 3 trial. Lancet Oncol. 2016;17(10):1426-1434.

20. Alden TD, Gianino JW, Saclarides TJ. Brain metastases from colorectal cancer. Dis Colon Rectum. 1996;39(5):541-545.

21. Damiens K, Ayoub JP, Lemieux B, et al. Clinical features and course of brain metastases in colorectal cancer: an experience from a single institution. Curr Oncol. 2012;19(5):254-258.

22. Benedix F, Kube R, Meyer F, et al. Comparison of 17,641 patients with right- and left-sided colon cancer: differences in epidemiology, perioperative course, histology, and survival. Dis Colon Rectum. 2010;53(1):57-64.

23. Nawa T, Kato J, Kawamoto H, et al. Differences between right- and left-sided colon cancer in patient characteristics, cancer morphology and histology. J Gastroenterol Hepatol. 2008;23(3):418-423.

24. Elsaleh H, Joseph D, Grieu F, Zeps N, Spry N, Iacopetta B. Association of tumour site and sex with survival benefit from adjuvant chemotherapy in colorectal cancer. Lancet. 2000;355(9217):1745-1750.

25. Miller RA. The aging immune system: primer and prospectus. Science. 1996;273(5271):70-74.

26. Utsuyama M, Hirokawa K, Kurashima C, et al. Differential age-change in the numbers of CD4+CD45RA+ and CD4+CD29+ T cell subsets in human peripheral blood. Mech Ageing Dev. 1992;63(1):57-68.

27. Grubeck-Loebenstein B. Changes in the aging immune system. Biologicals. 1997;25(2):205-208. 
28. Tamir A, Eisenbraun MD, Garcia GG, Miller RA. Age-dependent alterations in the assembly of signal transduction complexes at the site of T cell/APC interaction. J Immunol. 2000;165(3):1243-1251.

29. Catalano V, Loupakis F, Graziano F, et al. Mucinous histology predicts for poor response rate and overall survival of patients with colorectal cancer and treated with first-line oxaliplatin- and/or irinotecan-based chemotherapy. Br J Cancer. 2009;100(6):881-887.
30. Chen JS, Hsieh PS, Hung SY, et al. Clinical significance of signet ring cell rectal carcinoma. Int J Colorectal Dis. 2004;19(2): 102-107.

31. Yaeger R, Cowell E, Chou JF, et al. RAS mutations affect pattern of metastatic spread and increase propensity for brain metastasis in colorectal cancer. Cancer. 2015;121(8):1195-1203.

\section{Publish your work in this journal}

Cancer Management and Research is an international, peer-reviewed open access journal focusing on cancer research and the optimal use of preventative and integrated treatment interventions to achieve improved outcomes, enhanced survival and quality of life for the cancer patient. The manuscript management system is completely online and includes a very quick and fair peer-review system, which is all easy to use. Visit http://www.dovepress.com/testimonials.php to read real quotes from published authors. 